\title{
Resonance line shapes and catastrophes in particle-surface scattering
}

\author{
J. Margalef-Roig and S. Miret-Artés \\ Instituto de Matemáticas y Física Fundamental, Consejo Superior de Investigaciones Científicas, Serrano, 123, 28006 Madrid, Spain
}

(Received 22 June 2000; published 1 February 2001)

\begin{abstract}
In this work we present a general study of resonance line shapes within the catastrophe theory framework and valid for any type of scattering: particle-particle, particle-surface, or liquid and light-particle. The standard profiles analyzed, and issued from the multichannel scattering theory, correspond to isolated Fano-type, double, critical, and dipole resonances. They have been topologically classified according to the well-known elementary catastrophes: fold, cusp, and its dual and swallowtail, respectively. The onset of each structural change, or topological transition, at some external, critical parameter value, like the surface temperature, is discussed in terms of the probabilities of entering and leaving the resonance as well as of the direct scattering. Finally, atom-surface scattering is studied in more detail showing that the critical temperature is very close to the Debye surface temperature.
\end{abstract}

DOI: 10.1103/PhysRevB.63.094302

PACS number(s): 63.20.Dj

\section{INTRODUCTION}

Resonance line shapes and signatures have attracted a lot of interest in many branches of physics. Rules governing such intensity features have been sometimes reported but in many cases their applicability is very limited. One of the main reasons is due to the lack of a good knowledge of the interaction potential governing the physical process involved. However, the different line shapes of resonance features or, in mathematical terms, their different topologies found in experiments and calculations can be easily understood by a close examination of the nature of the critical point, that is, the resonance position. The theory available for such a study is the catastrophe or singularity theory (CT) introduced by Thom ${ }^{1}$ and widely developed by Zeeman ${ }^{2}$ (for an introduction see, for example, Refs. 3 and 4). CT is still being widely applied in different branches of physics such as, for example, thermodynamics for phase transitions, structural mechanics, aerodynamics, climate, quantum mechanics, and caustics and diffraction patterns. In particular, in atomic, molecular, and surface physics, the rainbow scattering, ${ }^{5}$ the asymptotic evaluation of integrals (uniform approximations), ${ }^{6}$ and the molecular geometry discussed in terms of the topology of the charge density ${ }^{7}$ are the three best known examples where CT has been successfully applied.

In most of those works, CT has not been applied in a rigorous way using all the concepts and theorems needed for its correct implementation. In particular, we study a mathematical property called transversality which guarantees that a given general function and its elementary catastrophe associated, expressed as a simple polynomial or canonical form, represents locally (around a critical point) up to a diffeomorphism such a function. Very recently, we have proposed and applied an algorithm based on the main theorems of CT to a standard phase transition problem. ${ }^{8,9}$ A big advantage of our approach is that provides a systematic way to analyze and classify the critical points of any general, analytical function susceptible to represent any physical process.

A catastrophe, in this context, would correspond to a coalescence of two or more critical points of the analytical function used to parametrize the different topologies observed in many theoretical calculations and/or experimental results. Obviously these topological manifestations are very common and have been observed many times but as far as we know they have not been recognized as such. Application of CT to resonances is quite straightforward since we are dealing with analytical functions depending on only one variable, usually the total energy (state variable, in CT terminology) and on several parameters as, for example, the temperature and the pressure (forming the so-called control space). In this control space is where caustics are defined. They are obtained from the bifurcation set which is calculated, for a one-dimensional problem, by equating to zero the first and second derivatives of a given function and eliminating the state variable. The corresponding parametric curves define the caustic.

After CT, the mathematical analysis begins with the calculation of the critical points $x_{0}$ of a given analytical function, $F(x)$. These points also called isolated or nondegenerate are calculated by the conditions $F^{\prime}\left(x_{0}\right)=0$ and $F^{\prime \prime}\left(x_{0}\right)$ $\neq 0$, where $F^{\prime}(x)$ and $F^{\prime \prime}(x)$ stand for the first and second derivatives of the function $F(x)$ with respect to $x$, respectively. These critical points will be called $k$ degenerate or nonisolated when the first $k$ derivatives are zero. The existence of degenerate critical points will indicate the onset of structural changes in a resonance profile when some external parameter is varied. The change of the profile is predicted to be abrupt when a given parameter crosses the caustic. Moreover, functions in the vicinity of these points are not structurally stable, that is, its qualitative properties (number and types of critical points) are changed by a sufficiently small perturbation of one or several parameters. Thus, in general, a $k$-fold critical degenerate point will split up into at most $k$ isolated critical points (possible fewer) by a perturbation which can be expressed, after CT, by a given canonical form.

A study of experimental resonance line shapes in terms of CT has been recently reported $^{10}$ in the context of atomsurface scattering for the system $\mathrm{He}-\mathrm{NaCl}(001)$. The corresponding selective adsorption resonance line shapes displaying mixed extrema structures (a Fano-type function) have been shown to be isomorph to the simplest elementary catastrophe, the fold catastrophe, when the surface temperature is varied around its Debye value. This type of functions is the 
simplest one where a coalescence of two isolated critical points can occur. Fano-type profiles in scattering processes appear when there is an interference between the resonance and background contributions. The former comes from the short-range attractive component of the potential and the later from its long-range component. On half-collision problems is the interference between the direct dissociation and the predissociation which provokes such profiles. Due to the fact that this interference behavior is very common in many physical processes, we are going to review it in a very general way and extend this study to three more interference mechanisms: double resonances, dipole resonances, ${ }^{11}$ and the so-called critical profiles recently observed in the scattering of $\mathrm{He}$ atoms from the $\mathrm{NaCl}(001)$ surface ${ }^{12}$ and $D_{2}$ molecules from the $\mathrm{Cu}(001)$ surface, ${ }^{13}$ the originally predicted resonance mechanisms leading to such singular behaviors are called the critical kinematic (CK) (Ref. 14) and focussed inelastic resonance (FIR) (Refs. 15 and 16) effects. We will finally show that the critical temperature for the topological transition should be very close to the corresponding surface Debye temperature. The theoretical starting point will be the multichannel resonance scattering.

\section{GENERALITIES ABOUT CATASTROPHE THEORY WITH ONE STATE VARIABLE: THE CT ALGORITHM}

In this section we are going to review very briefly the concepts and theorems of CT used thorought this work. For simplicity, we will focus on one dimensional functions with several parameters. CT deals with the singularities of smooth real-valued functions. The character of the singularity is revealed by perturbing locally the function around such a point. If, as a result of a perturbation, the qualitative properties of the function remain unaffected, we will say that this function is stable or structurally stable. A very important concept is the so-called $k$ jet of a given function at a given point which is defined as its Taylor series truncated beyond terms of degree $k$. Now the next important step is to know what information is lost when we truncate the Taylor series of a function around a given point, namely, the problem of determinacy. In other words, we are interested in determining whether a function can be truncated and if so, up to what degree the Taylor expansion can be truncated without any loss of substantial information. In this way, we determine the most general family of functions (unfolding) of minimum dimension (the least number of Taylor series coefficients) which contains the original function. The unfolding dimension is the number of parameters describing a general perturbation and the minimum number to describe it is called the codimension. When all the unfolding parameters go to zero, the remainder of the universal unfolding is called the germ of the canonical form.

When the $k$ jets of two functions are equal we say that both functions are $k$ equivalent. A germ $g$ is $k$ determinate if for every germ $f$ such that both $k$ jets are equal we have that $f$ and $g$ are equivalent (or related by a change of coordinates, being usually expressed as $f \sim g$ ). The determinacy of a germ $g$ is the smallest natural number $k$ such that $g$ is $k$ determi- nate and this number is called the determinative number, $\sigma(g)$. A sufficient algebraic condition for the $k$ determinacy of $g$ can be written as

$$
\langle x\rangle^{k+1} \subset\langle x\rangle^{2} \Delta(g)+\langle x\rangle^{k+2},
$$

where $\Delta(g)$ is the ideal of Jacobi of the germ $g$ which is defined for one variable by

$$
\Delta(g)=\left\langle\frac{d g}{d x}\right\rangle
$$

and the notation $\langle\cdots\rangle$ is used to denote the ideal of the ring formed by the set of germs in one dimension, $E(1)$. The powers in Eq. (1) are interpreted operationally as

$$
\langle x\rangle^{n}=\left\{x^{n} \cdot f / f \in E(1)\right\}, \text { for every } n \in \mathbb{N} .
$$

The next step is to introduce the concept of transversality as a means to study structural stability and genericity. The transversality condition is not widely used in the literature to classify physical phenomena in terms of elementary catastrophes. When a property of a given function is invariant under a perturbation, this property is called generic or structurally stable. One of the main theorems about $k$ transversality for unfoldings can be stated as follows: Let us consider a germ $g$ $k$ determinate and $f$ and $h$ two unfoldings of $g$ with $r$ parameters which are $k$-tranversal; then $f$ and $h$ are isomorphic. Moreover, if $h$ is an unfolding of $g$, the algebraic condition for the transversality can be established as follows: we say that $h$ is $k$ transversal if

$$
\langle x\rangle=\Delta(g)+\langle x\rangle^{k+1}+V_{h},
$$

where $V_{h}$ is the real vector space generated by the vectors $D_{\lambda_{1}} g(x, 0)-D_{\lambda_{1}} g(0,0), \ldots, D_{\lambda_{r}} g(x, 0)-D_{\lambda_{r}} g(0,0)$ where $D_{\lambda}$ stands for the partial derivative with respect to the $\lambda$ parameter.

After this brief introduction, the CT program or algorithm applied $^{8,9}$ can be now established as: Let $F(x, \lambda)$ be a real function with $x \in \mathbb{R}$ the state variable and control parameters $\lambda_{1}, \ldots, \lambda_{r}\left(\lambda \in \mathbb{R}^{r}\right)$; that is, $F: \mathbb{R}^{1+r} \rightarrow \mathbb{R}$. Then we proceed as follows:

(i) We pick $\left(x_{0}, \lambda_{0}\right)$ such that $x_{0}$ is a degenerate critical point of $F(x, \lambda)$ and we build the unfolding $h(x, \lambda)=F(x$ $\left.+x_{0}, \lambda+\lambda_{0}\right)-F\left(x_{0}, \lambda_{0}\right)$ and obtain the germ as $g(x)$ $=h(x, 0)$. Doing this, we have translated this local study to the origin of coordinates.

(ii) One calculates the determinacy and codimension of $g$ from the $k$ jet of $g$. Of course, if $g$ is $k$ determinate then $g$ $\sim j^{k}(g)$, that is, the function $g$ is equal to $j^{k}(g)$ up to a change of coordinates and hence they are equivalent and have qualitatively the same properties. Moreover, their codimensions are equal; that is, $\operatorname{cod}(g)=\operatorname{cod}\left[j^{k}(g)\right]$.

(iii) One studies after the $k$ transversality of $h$ and, if this function is $k$ transversal, we can affirm that $h$ and the canonical unfolding of $g$ are isomorph. Thus we can replace the original $h$ unfolding by that canonical unfolding. If not, we can claim that the $F$ function is not susceptible to be studied by CT. 


\section{STANDARD RESONANCE LINE SHAPES IN MULTICHANNEL SCATTERING}

Our starting point is the multichannel resonance scattering theory as formulated by Ref. 17 . The total probability $P_{t}$ of the transition from the incident channel $i$ to a final channel $f$, via an isolated resonance, is given by the square modulus of the collision $S$ matrix elements

$$
P_{t}=\left|S_{f i}\right|^{2}=\left|S_{b, f i}+i \frac{B_{f i}}{x+i}\right|^{2},
$$

with $S_{b, f i}$ being the collision background $S$ matrix, which includes all elastic and inelastic contributions except for those coming from the resonance. Its square modulus gives the probability of the direct, i.e., nonresonant, scattering, $P_{b}=\left|S_{b, f i}\right|^{2}$. The matrix element $B_{f i}$ is such that its square modulus $\left|B_{f i}\right|^{2}=\sigma^{2}$ links the probabilities of entering $\left(P_{\text {in }}\right)$ and leaving $\left(P_{\text {out }}\right)$ the bound state according to

$$
\sigma^{2}=4 \cdot P_{\text {out }} \cdot P_{\text {in }} \cdot
$$

This matrix element determines the signature of the resonance profile. ${ }^{14,15}$ In general, $P_{\text {out }}$ and $P_{\text {in }}$ are equal except when the time-invariance property does not hold. Both probabilities can be treated independently according to the independence hypothesis widely accepted in scattering theory which states that any scattering event can be separated in two steps: formation of the resonance with probability $P_{\text {in }}$ and its ulterior decay with probability $P_{\text {out }}$. In Eq. (5), we have introduced a dimensionless variable $x$, which can be expressed in terms of the resonance position $\bar{E}$ and width $\Gamma$ as

$$
x=(2 / \Gamma) \cdot(E-\bar{E}) .
$$

Thus $x=0$ gives the resonance position and $x= \pm 1$ the position shifted by $\pm \Gamma / 2$, covering the resonance region.

Any matrix element from Eq. (5) can also be rewritten in terms of an analytical function $F_{1}(x)$ as

$$
F_{1}(x)=\alpha+\beta x+\frac{\sigma^{2}+2 \rho(\cos \phi-x \sin \phi)}{x^{2}+1} .
$$

Here, it has been assumed that the background contribution to the resonance profile is a smooth function of $x$, that is, $P_{b}=\left|S_{b, f i}\right|^{2}=\alpha+\beta x$; and $b_{r}=\rho \cos \phi$ and $b_{i}=\rho \sin \phi$ are the real and imaginary parts of the product $B_{f i} \cdot S_{b, f i}^{*}$, respectively. In particular, the square modulus of this complex number is related to the scattering probabilities by the following expression:

$$
\left|B_{f i} \cdot S_{b, f i}^{*}\right|^{2}=\rho^{2}=4 \cdot P_{\text {out }} \cdot P_{\text {in }} \cdot P_{b} .
$$

As we will show later, the interference between the background and resonant contributions can give place to the coalescence of the two critical points (Fano-type profile) displayed by Eq. (8).

Another mechanism of coalescence of critical points is the so-called double resonance which is also very common in scattering problems. In general, we could envisage the interference of more than two resonances. However, we will il- lustrate here how to proceed for the simplest case where only two resonances interfere. The profile is given by

$$
P_{t}=\left|S_{f i}\right|^{2}=\left|S_{b, f i}+i \frac{B_{1, f i}}{x_{1}+i}+i \frac{B_{2, f i}}{x_{2}+i}\right|^{2},
$$

with the $x_{1}$ and $x_{2}$ variables defined by

$$
x_{1}=\left(2 / \Gamma_{1}\right) \cdot\left(E-\bar{E}_{1}\right)
$$

and

$$
x_{2}=\left(2 / \Gamma_{2}\right) \cdot\left(E-\bar{E}_{2}\right) .
$$

To facilitate the study of this new case we need to introduce two different variables,

$$
\begin{gathered}
2 \Delta=x_{2}-x_{1}, \\
x=x_{1}+\Delta=x_{2}-\Delta,
\end{gathered}
$$

and any matrix element of Eq. (10) can be rewritten as

$$
\begin{aligned}
F_{2}(x)= & \alpha+\beta x+\frac{\sigma_{1}^{2}+2 \rho_{1}\left[\cos \phi_{1}-(x+\Delta) \sin \phi_{1}\right]}{(x+\Delta)^{2}+1} \\
& +\frac{\sigma_{2}^{2}+2 \rho_{2}\left[\cos \phi_{2}-(x-\Delta) \sin \phi_{2}\right]}{(x-\Delta)^{2}+1} \\
& +\frac{\left(x^{2}-\Delta^{2}+1\right) 2 \varrho \cos \varphi-4 \Delta \varrho \sin \varphi}{\left(x^{2}-\Delta^{2}+1\right)^{2}+4 \Delta^{2}}
\end{aligned}
$$

where the symbols, $\sigma, \rho$, and $\phi$ are defined for each resonance with a subindex 1 or 2 , respectively, and the new symbols $\varrho$ and $\varphi$ are used for the modulus and phase of the complex number $B_{1, f i} \cdot B_{2, f i}^{*}$, respectively.

The isolated resonance case above described is also called sometimes monopole resonance. In scattering theory, it is possible to describe from a mathematical point of view what it is called in general multipole resonances. ${ }^{11}$ The existence of a dipole resonance was first reported in particle physics. This case has a lot of similarities to the double resonance but, as we will see, its topological manifestations are not strictly the same. We are going also to analyze this profile. This profile can be expressed as

$$
P_{t}=\left|S_{f i}\right|^{2}=\left|S_{b, f i}+2 i x \frac{B_{f i}}{(x+i)^{2}}\right|^{2},
$$

which after a little algebra any matrix element becomes the following analytical function:

$$
\begin{aligned}
F_{3}(x)= & \alpha+\beta x \\
& +4 \frac{x \rho \sin \phi+\left(\sigma^{2}+2 \rho \cos \phi\right) x^{2}-x^{3} \rho \sin \phi}{\left(x^{2}+1\right)^{2}},
\end{aligned}
$$

where the parameters have the same meaning as before.

In atom-surface scattering, there are special resonance profiles called critical profiles where the function $F_{1}(x)$ is replaced by 


$$
F_{4}(x)=\alpha+\beta x+\frac{\sigma^{2}+2 \rho\left(\cos \phi-x^{2} \sin \phi\right)}{x^{4}+1} .
$$

These profiles occur under special incident conditions of the incoming particle scattering (elastically or inelastically) from a given surface. The $\mathrm{CK}$ and FIR mechanisms were predicted $^{14,15}$ and recently observed in the scattering of $\mathrm{He}$ atoms and $D_{2}$ molecules from insulators and metal surfaces, ${ }^{12,13}$ respectively.

Equations (8), (14), (16), and (17) defining the analytical functions $F_{i}(x)$ with $i=1,2,3,4$ are the starting point for the topological analysis of resonance profiles by means of CT.

\section{APPLICATION OF THE CT PROGRAM}

\section{A. Isolated resonances: Fano-type profiles}

The background contribution $\left[P_{b}=\alpha+\beta x\right.$ in Eq. (8)], taken as a small perturbation near the resonance region, affects differently the line shapes around the isolated and nonisolated critical points. If the coefficients accompanying $x$ in Eq. (8) are equal to zero $(\beta=0$ and $\sin \phi=0)$, then $x=0$ is not a degenerate critical point. At these conditions the resonance profile $F_{1}(x)$ will exhibit a symmetric Lorentzian function and show (i) a maximum whenever $P_{b}<P_{\text {out }} \cdot P_{\text {in }}$ and (ii) a minimum whenever $P_{b}>P_{\text {out }} \cdot P_{\text {in }}$. These profiles are structurally stable from a topological point of view. Moreover, if $\sin \phi=0$ only, asymmetric maxima or minima will be exhibited depending on the relation among the abovementioned scattering probabilities.

On the contrary, when $\beta \neq 0$ and $\sin \phi \neq 0$, asymmetric Fano-type functions (minimum-maximum structures) are present. We are interested under what conditions the two isolated critical points coalesce. A close examination of the three first derivatives reveals very important consequences. Thus we have that

(i) If $F_{1}^{\prime}(0)=0$ then $\beta=2 \rho \sin \phi$, that is, the coefficients accompanying $x$ are equal [see Eq. (8)]. For convenience, we write this condition as $\delta_{1}=2 \rho \sin \phi / \beta=1$.

(ii) If $F_{1}^{\prime \prime}(0)=0$ then $\cos \phi=-\left(\sigma^{2} / 2 \rho\right)$ or $\delta_{2}$ $=2 \rho \cos \phi / \sigma^{2}=-1$ which implies that $P_{b}>P_{\text {out }} \cdot P_{\text {in }}$. Both conditions can be written in a single expression such $4 \rho^{2}$ $=\beta^{2}+\sigma^{4}$.

(iii) Finally, if $F_{1}^{\prime \prime \prime}(0) \neq 0$ then $\sin \phi \neq 0$ and $\beta \neq 0$.

These three requirements form a necessary condition for the most elementary catastrophe, that is, the fold catastrophe. The bifurcation set can be obtained by equating to zero the first and second derivatives of $F_{1}(x)$ and, from both equations, to eliminate the state variable $x$. In doing so, we have that the bifurcation set is

$$
B_{F_{1}} \equiv \delta_{2}=(\beta \sqrt{3} / 9) \cdot\left(\delta_{1}+8\right) \cdot \sqrt{1-\delta_{1}},
$$

with $\delta_{1} \leqslant 1$. In general, the bifurcation set is not easy to extract from a general function since the $x$ variable can be involved in a quite complex way (for example, transcendental functions).
From this preliminary study we can say that the function $F_{1}(x)$ is susceptible to be studied by CT. We proceed therefore to apply the CT program. We start building the unfolding of $F_{1}(x)$ as

$$
\begin{aligned}
h(x, \beta, \sigma, \rho, \phi)= & F_{1}\left(x+x_{0}, \beta+\beta_{0}, \sigma+\sigma_{0}, \rho+\rho_{0}, \phi+\phi_{0}\right) \\
& -F_{1}\left(x_{0}, \beta_{0}, \sigma_{0}, \rho_{0}, \phi_{0}\right)
\end{aligned}
$$

with the germ defined by

$$
g(x)=h(x, 0,0,0,0)
$$

and where the critical point has been shifted to the origin of coordinates, the $\alpha$ parameter not being relevant in this CT analysis. Now we impose the conditions at $x_{0}=0$,

$$
g^{\prime}(0)=g^{\prime \prime}(0)=0, \quad g^{\prime \prime \prime}(0) \neq 0,
$$

which lead to the system of equations

$$
\begin{gathered}
0=\beta_{0}-2 \rho_{0} \sin \phi_{0}, \\
0=\sigma_{0}^{2}+2 \rho_{0} \cos \phi_{0}, \\
0 \neq 12 \rho_{0} \sin \phi_{0}
\end{gathered}
$$

with $\rho_{0}>0$ and $\left.\phi_{0} \in\right] \pi / 2, \pi[\cup] \pi, 3 \pi / 2[$. Then the three jet of the germ $g$ is

$$
j^{3}(g)=2 x^{3} \rho_{0} \sin \phi_{0}
$$

and the ideal of Jacobi of the germ is

$$
\Delta(g)=\left\langle g^{\prime}(x)\right\rangle=\left\langle x^{2}\right\rangle
$$

The codimension of the germ is the dimension of the quotient vector space, $\operatorname{cod}(g)=\operatorname{dim}\langle x\rangle /\left\langle x^{2}\right\rangle=1$ and a basis of this vector space is $\{[x]\}$. After Eq. (1), the germ $g$ is three determinate. Then

$$
j^{3}(g) \sim g \sim z^{3}
$$

and the canonical form

$$
z^{3}+\lambda_{1} z
$$

is a $k$-transversal unfolding of $z^{3}$ for $k \geqslant 3$. This canonical form corresponds to the fold elementary catastrophe.

Next, the important point is to show that the $F_{1}(x)$ function and the canonical form Eq. (26) for the fold catastrophe are isomorph. When this is true, we can affirm that both functions are strictly equal and present the same topological properties as well as describe the same scattering process. As said in the Introduction, for this goal, a mathematical local property called transversality, Eq. (4), has to be proved, ${ }^{2}$ namely

$$
\langle x\rangle=\left\langle x^{2}\right\rangle+\langle x\rangle^{3+1}+V_{h},
$$

where $V_{h}$ is the vector space of the transversality and reads in our case as 


$$
\begin{aligned}
V_{h}= & \left\langle x, x 2 \sigma_{0} \frac{-2 x_{0}}{\left(x_{0}^{2}+1\right)^{2}} \ldots, x\left[-\left(x_{0}^{2}+1\right)^{-1} 2 \sin \phi_{0}\right.\right. \\
& -4 x_{0}\left(\cos \phi_{0}-x_{0} \sin \phi_{0}\right)\left(x_{0}^{2}+1\right)^{-2}+\ldots, \\
& x\left[-\left(x_{0}^{2}+1\right)^{-1} 2 \rho_{0} \cos \phi_{0}+4 x_{0} \rho_{0}\left(\sin \phi_{0}+x_{0} \cos \phi_{0}\right)\right. \\
& \left.\left.\left.\times\left(x_{0}^{2}+1\right)^{-2}\right]+\cdots\right]\right\rangle_{\mathbb{R}}
\end{aligned}
$$

where after deriving with respect to the parameters $\beta, \sigma, \rho$, and $\phi$, the resulting functions depending on $x$ are replaced by the first terms of their Taylor series. Due to the fact that $V_{h}$ contains the generator $x$ the transversality condition applied to $h$, Eq. (4), is fulfilled for $k=3$. Thus $F_{1}(x)$ is three transversal. Therefore, as this function has four parameters and the canonical form of the fold catastrophe only one, there are three parameters which are irrelevant. Finally we can claim that $F_{1}(x)$ and the canonical unfolding

$$
\Gamma_{1}\left(z, \lambda_{1}, \lambda_{2}, \lambda_{3}, \lambda_{4}\right)=z^{3}+\lambda_{1} z
$$

are isomorph; that is, there are three changes of coordinates and a perturbation of parameters involving the state variable and the parameters of the theory. CT guarantees these three changes of coordinates, however, it can be in general very difficult to find them.

The bifurcation set of Eq. (29) is

$$
B_{\Gamma_{1}} \equiv \lambda_{1}=0
$$

and is related to the bifurcation set of $F_{1}(x)$ by

$$
B_{F_{1}}=B_{\Gamma_{1}} \times \mathrm{R}^{3} .
$$

$F_{1}$ and $\Gamma_{1}$ present the same topological properties and $\Gamma_{1}$ can be used to describe exactly the same physical phenomenon. It is well-known from CT (Refs. 3 and 4) that the fold catastrophe has the following topological properties: its bifurcation set is a single point $\left[\lambda_{1}=0\right.$ in Eq. (29) $]$ and when the physical problem passes through this value an abrupt change in the topology (topological transition) occurs. Again, from CT, it follows that for negative values of $\lambda_{1}$, $F_{1}(x)$ has two critical points (a maximum and minimum), while for positive $\lambda_{1}$ values, no critical point is found in $F_{1}(x)$. In the bifurcation, $F_{1}(x)$ will display a plateau around the resonance position. These three different topologies can be found in any standard book of $\mathrm{CT}^{3,4}$ In summary, when the background contribution is not negligible and $P_{b}>P_{\text {in }} \cdot P_{\text {out }}$ an abrupt change in the resonance profile is expected by varying an external parameter as, for example, the temperature of the surface in atom-surface scattering. ${ }^{10}$

\section{B. Double resonances}

The unfolding of $F_{2}(x)$ is

$$
h(x, \beta, \Delta)=F_{2}\left(x+x_{0}, \beta+\beta_{0}, \Delta+\Delta_{0}\right)-F_{2}\left(x_{0}, \beta_{0}, \Delta_{0}\right)
$$

and the germ defined again by

$$
g(x)=h(x, 0,0),
$$

where for simplicity we have assumed that only the $\beta$ and $\Delta$ parameters play an important role in this CT analysis. Now we impose the conditions at $x_{0}=\Delta_{0}=0$,

$$
g^{\prime}(0)=g^{\prime \prime}(0)=g^{\prime \prime \prime}(0)=0, \quad g^{(i v)}(0) \neq 0,
$$

which lead to the system of equations

$$
\begin{gathered}
0=-\beta_{0}+2\left(\rho_{1} \sin \phi_{1}+\rho_{2} \sin \phi_{2}\right), \\
0=\sigma_{1}^{2}+\sigma_{2}^{2}+2\left(\rho_{1} \cos \phi_{1}+\rho_{2} \cos \phi_{2}+\varrho \cos \varphi\right), \\
0=2\left(\rho_{1} \sin \phi_{1}+\rho_{2} \sin \phi_{2}\right), \\
0 \neq 24(2 \varrho \cos \varphi-1)
\end{gathered}
$$

and therefore $\beta_{0}=0$. Then the four jet of the germ $g$ is

$$
j^{4}(g)=\frac{1}{24} g^{(i v)}(0) x^{4}
$$

and the ideal of Jacobi of the germ is

$$
\Delta(g)=\left\langle g^{\prime}(x)\right\rangle=\left\langle x^{3}\right\rangle
$$

Finally the codimension of the germ is the dimension of the quotient vector space, $\operatorname{cod}(g)=\operatorname{dim}\langle x\rangle /\left\langle x^{3}\right\rangle=2$ and a basis of this vector space is $\left\{[x],\left[x^{2}\right]\right\}$. After Eq. (1), the germ $g$ is four determinate and

$$
j^{4}(g) \sim g \sim \pm z^{4}
$$

since $g^{(i v)}(0)$ can be positive or negative. The canonical forms

$$
\pm z^{4}+\lambda_{1} z+\lambda_{2} z^{2}
$$

are $k$-transversal unfoldings of $\pm z^{4}$ for $k \geqslant 4$. These canonical forms correspond to the cusp (plus sign) elementary catastrophe and its dual (minus sign). The transversality now reads

$$
\langle x\rangle=\left\langle x^{3}\right\rangle+\langle x\rangle^{4+1}+V_{h},
$$

where $V_{h}$ is the vector space of the transversality, this time deriving only with respect to the parameters $\beta$ and $\Delta$. This condition is fulfilled for $k=4$. The canonical unfoldings of the cusp catastrophe and its dual,

$$
\Gamma_{2}\left(z, \lambda_{1}, \lambda_{2}\right)= \pm z^{4}+\lambda_{1} z+\lambda_{2} z^{2},
$$

are isomorph to $F_{2}(x)$; that is, there are four changes of coordinates and a perturbation of parameters involving the state variable and the parameters of the theory.

The bifurcation set of Eq. (41) is

$$
B_{\Gamma_{2}} \equiv 27 \lambda_{1}^{2}+8 \lambda_{2}^{3}=0
$$

and is related to the bifurcation set for $F_{2}(x)$ by

$$
B_{F_{2}}=B_{\Gamma_{2}}
$$

As before, both unfoldings present exactly the same topological properties and $\Gamma_{2}$ can be used to describe the same 
physical phenomenon. It is well-known from CT (Refs. 3 and 4) that the cusp catastrophe and its dual exhibit the following topological properties: bimodality (two minima for the cusp or two maxima for the dual cusp) when the control parameters lie within the cusp-shaped area, divergence of the linear response since two nearby paths in control space may lead to widely different topological features and hysteresis which occurs when a physical process is not reversible. This final aspect will be discussed in more detail in next section.

\section{Dipole resonances}

The procedure applied to the function $F_{3}(x)$ is completely similar to the preceding cases. Due to the fact that this function is slightly more complicated the calculation of the succesive derivatives is much more tedious. Again, we build the unfolding of $F_{3}(x)$ as before,

$$
\begin{aligned}
h(x, \beta, \sigma, \rho, \phi)= & F_{3}\left(x+x_{0}, \beta+\beta_{0}, \sigma+\sigma_{0}, \rho+\rho_{0}, \phi+\phi_{0}\right) \\
& -F_{3}\left(x_{0}, \beta_{0}, \sigma_{0}, \rho_{0}, \phi_{0}\right),
\end{aligned}
$$

with the germ defined by

$$
g(x)=h(x, 0,0,0,0) .
$$

Now we impose again the following conditions:

$$
g^{\prime}(0)=g^{\prime \prime}(0)=g^{\prime \prime \prime}(0)=0,
$$

giving rise to the system of equations

$$
\begin{gathered}
0=\beta_{0}+4 \rho_{0} \sin \phi_{0}, \\
0=8\left(\sigma_{0}^{2}+2 \rho_{0} \cos \phi_{0}\right), \\
0=-72 \rho_{0} \sin \phi_{0}
\end{gathered}
$$

with $\beta_{0}=0, \sin \phi_{0}=0$. With these values, the fourth derivative is identically zero and the fith derivative is nonzero. Then, the five jet of the germ is equivalent to

$$
j^{5}(g) \sim g \sim z^{5},
$$

$g$ is five determinate, its codimension is 3 and its ideal of Jacobi is

$$
\Delta(g)=\left\langle g^{\prime}(x)\right\rangle=\left\langle x^{4}\right\rangle .
$$

The canonical form

$$
z^{5}+\lambda_{1} z+\lambda_{2} z^{2}+\lambda_{3} z^{3}
$$

is a $k$-transversal unfolding of $z^{5}$ for $k \geqslant 5$. This canonical form corresponds to the swallowtail elementary catastrophe. For the transversality we have shown that Eq. (4) is fulfilled for $k=5$. In $V_{h}$ derivation is with respect to the parameters $\beta, \sigma, \rho$, and $\phi$, replacing the resulting functions by the first terms of their Taylor series. Then $F_{3}(x)$ and the canonical unfolding,

$$
\Gamma_{3}\left(z, \lambda_{1}, \lambda_{2}, \lambda_{3}, \lambda_{4}\right)=z^{5}+\lambda_{1} z+\lambda_{2} z^{2}+\lambda_{3} z^{3},
$$

are isomorph but only two of the four parameters are relevant. The bifurcation set of Eq. (51) is much more compli- cated and can be found in any standard book of CT. ${ }^{3,4}$ The bifurcation set for $F_{3}(x)$ is related to that of $\Gamma_{3}$ by

$$
B_{F_{3}}=B_{\Gamma_{3}} \times \mathbb{R}^{2} .
$$

The topological properties are much more complicated and the interested reader is again referred to any standard book about CT.

\section{Critical resonances}

The unfolding of $F_{4}(x)$ is

$$
\begin{aligned}
h(x, \beta, \sigma, \rho, \phi)= & F_{4}\left(x+x_{0}, \beta+\beta_{0}, \sigma+\sigma_{0}, \rho+\rho_{0}, \phi+\phi_{0}\right) \\
& -F_{4}\left(x_{0}, \beta_{0}, \sigma_{0}, \rho_{0}, \sigma_{0}\right)
\end{aligned}
$$

and the germ

$$
g(x)=h(x, 0,0,0,0) .
$$

Now we impose the same conditions at $x_{0}=0$,

$$
g^{\prime}(0)=g^{\prime \prime}(0)=g^{\prime \prime \prime}(0)=0, \quad g^{(i v)}(0) \neq 0,
$$

which lead to $\beta_{0}=0$ and $\sin \phi_{0}=0$ as before. Then the four jet of the germ $g$ is

$$
j^{4}(g)=\frac{1}{24} g^{(i v)}(0) x^{4}=-\left(\sigma_{0}^{2}+2 \rho_{0}\right) x^{4}
$$

and the same ideal of Jacobi of the germ is reached as in the double resonance case. The codimension of the germ is 2 , it is four determinate and

$$
j^{4}(g) \sim g \sim \pm z^{4}
$$

A basis is again $\left\{[x],\left[x^{2}\right]\right\}$. The canonical forms are again those of the cusp and its dual. $F_{4}(x)$ has been proved that it is also four transversal and the canonical unfolding,

$$
\Gamma_{4}\left(z, \lambda_{1}, \lambda_{2}, \lambda_{3}, \lambda_{4}\right)= \pm z^{4}+\lambda_{1} z+\lambda_{2} z^{2},
$$

is isomorph to $F_{4}(x)$. The corresponding bifurcation set is the same than for the canonical unfolding $\Gamma_{2}$,

$$
B_{\Gamma_{4}} \equiv B_{\Gamma_{2}},
$$

and is related to the bifurcation set for $F_{4}(x)$ by

$$
B_{F_{4}}=B_{\Gamma_{4}} \times \mathbb{R}^{3} .
$$

\section{PHYSICAL DISCUSSION AND CONCLUSIONS}

This kind of study has been initially tackled in atomsurface scattering where clear evidences of some of the singular behaviors found here have been already observed. ${ }^{10,12,13}$ Our main goal in this work has been to extend, justify, and generalize that previous topological analysis to the most common resonance features reported in many physical processes. We have shown mathematically that resonance line shapes are governed by $\mathrm{CT}$, the different topologies being dictated only by the canonical forms. In particular, the analytical functions $F_{1}(x)$ and $F_{2}(x)$ representing Fano-type and double resonance profiles, respectively, 
are isomorph to the most elementary (lower codimension) catastrophes; that is, the fold and cusp catastrophes. The dipole resonance has been analyzed in some detail since it points out a tendency about how to reach higher codimensions. Moreover, the critical profiles provide a good illustration of the selective adsorption phenomenon, very well known in the context of particle-surface scattering, and where the corresponding CK and FIR effects supply a means to make weak resonance features visible when metal surfaces are probed. In fact, the $F_{4}(x)$ function has been successfully applied to fit the resonance line shape observed in the scattering of $D_{2}$ molecules from the $\mathrm{Cu}(001)$ surface and showed the topological transition around the critical temperature predicted in this work. ${ }^{13}$ Even more, from that fitting, the lifetime of the corresponding resonance has been easily obtained. This is a very important issue since many times it is very difficult to extract (inelastic or elastic) resonance lifetimes if no information about the topologies of line shapes is known. In this sense, the present study can be considered as a useful guide for experimentalists. The final conclusions drawn in this work can also be extended to half collision problems, nuclear scattering, neutron-surface (or liquid) scattering, electron-molecule scattering, gas-phase scattering, etc.

A second aspect of this general study has consisted of stressing the importance of the linear behavior displayed by the background contribution. In other words, this theoretical development is based on two main assumptions: the background contribution inside the resonance region can be considered as a small perturbation and, at least, a linear term in energy should be included to fulfill the property of transversality. Obviously, resonance processes very near to threshold conditions or with very active backgrounds are not good examples to apply such a study.

Interestingly enough is the interpretation we can give to the bifurcation sets or caustics found. In optics, for example, the envelope of rays reflected from a curved surface forms a caustic; two or more rays coalesce on each point of the caustic and the intensity along the envelope is very high. A completely different meaning should be attributed to caustics issued from resonance processes. By passing through the bifurcation set the topology of the profile is changed abruptly; it delimits in the control space the borders where a topological transition takes place. As has been established, the different line shapes predicted by CT are related to the behavior of the background which can be modified in several ways. First, depending on the $S$-matrix element chosen to describe a given resonance, the arrangement of the collisional channels determines drastically such a behavior. Second, any external parameter such as the temperature of the target, the initial orientation of the collisional partners, the pressure in gas phase or liquid scattering, the intensity of the radiation field in a half collision problem, etc., can provoke substantial modifications on the background contribution and it would be possible to follow continuously the topological transitions predicted by CT for a given resonance. And, third, each line shape displayed is associated to a given relation among the three fundamental probabilities discussed in the text, $P_{\text {in }}, P_{\text {out }}$, and $P_{b}$. Moreover, in some cases, as for example in the critical profiles, the density of resonance states becomes infinity ${ }^{18}$ In the double resonance case, the same interpretation could be considered.

Along these lines, it has been clearly established that CT describes the behavior of a system by comparing it to certain patterns, the canonical forms. However, in most cases try to find the changes of coordinates leading to such canonical polynomials is indeed impossible but their existence is assured by CT. In this sense, the procedure followed in this work is not sufficient to give an interpretation of the microscopic phenomena which bring about the critical behavior. For example, in atom/diatom-surface scattering, the variation of the surface temperature has been recently shown to produce topological transitions for a given selective adsorption resonance. ${ }^{10,13}$ In both cases, it was remarkable that the critical temperatures observed were very close to their corresponding surface Debye temperatures where multiphonon processes begin to be important. We feel that this fact is not a coincidence at all but, unfortunately, we also think that CT is not in a position to provide a complete theoretical justification of the underlying physical mechanism. The temperature dependence of $P_{b}, P_{\text {in }}$, and $P_{\text {out }}$ is really very different if elastic or inelastic (phonon-assisted) resonances are involved. Nevertheless, for elastic selective adsorption resonances, some behaviors can be easily devised. For example, $P_{b}$ is expected to follow the overall Debye-Waller attenuation. The parameter $\sigma$, involving the "in" and "out" resonant probabilities, should depend weakly on the temperature since these probabilities are ratios between a partial width (resonance decay by an open diffraction channel) and the total width (resonance decay by all open diffraction channels) of the resonance. In contrast, the parameter $\rho^{2}$ expressed in terms of the background probability should also follow the thermal Debye-Waller (DW) attenuation and be the major contribution to the surface temperature of the numerators from the functions $F_{i}(x)$ with $i=1,2,3,4$. In a very good approximation, the $x$ variable is independent on the surface temperature. On the other hand, it is well known that the DW exponent depends linearly on the surface temperature, the proportional factor being a function mainly of the scattering geometry and masses. On the other hand, this exponent is usually interpreted as the average number of phonons exchanged in the collision. Thus, if we admit that such an exponent is close to 1 , a more or less limit for single phonon processes, the critical temperature will be in a very good approximation close to the surface Debye temperature whenever the proportional factor is also close to 1 . This is exactly what has been observed recently in Ref. 10.

Concerning the hysteresis problem which arises in Sec. IV B where the cusp catastrophe appears, several comments need to be mentioned. It is well known that hysteresis occurs whenever a physical process is not strictly reversible. Moreover, hysteresis is also very much related to the conventions used in CT. The bifurcation set is associated to the so-called delay convention, valid when the values of the control parameters are changing with time very slowly. On the contrary, in the other extreme, for the so-called Maxwell or conflicting set, the Maxwell convention is applied. In this new set, the critical values of a function at two or more critical 
points are degenerate and the equations determining it are called the Clausius-Clapeyron equations. It is well known that when a physical system exhibits large fluctuations, the Maxwell convention holds and hysteresis fails to occur. Thus, in physical irreversible resonance processes, the presence of double or critical resonances is a indirect indication that hysteresis should exist. In atom-surface scattering, for example, when the surface is heated the response to the diffraction of He atoms is different from that observed when the surface is cooled down. The same is predicted in the selective adsorption phenomenon.

The last comment is concerning the isomorphy. In this work, we have clearly established that the study of the transversality is fundamental in order to be sure that the function describing the physical process is indeed isomorphic to one of the elementary catastrophes. As has been said, this aspect is many times neglected in applying CT to different branches of physics and chemistry. Moreover, thanks to that mathematical property, completely different phenomena are closely related among them due to the genericity underlying in nature, being remarkably described and justified in the CT framework.

\section{ACKNOWLEDGMENTS}

This work has been supported by DGICYT (Spain) under Contract Nos. PB96-0651-C03-01 and PB95-0071 and the European Contract No. HPRN-CT-1999-00005.
${ }^{1}$ R. Thom, Stabilité Structurelle et Morphogénese (Benjamin, New York, 1972).

${ }^{2}$ E. C. Zeeman, Catastrophe Theory, Selected Papers 1972-1977 (Addison-Wesley, Reading, MA, 1977).

${ }^{3}$ T. Poston and I. Stewart, Catastrophe Theory and its Applications (Pitman, London, 1978).

${ }^{4}$ R. Gilmore, Catastrophe Theory for Scientists and Engineers (Dover, New York, 1981).

${ }^{5}$ M. V. Berry, J. Phys. A 8, 566 (1975); Adv. Phys. 25, 1 (1976).

${ }^{6}$ J. N. L. Connor, Mol. Phys. 31, 33 (1976).

${ }^{7}$ R. F. W. Bader, Atoms in Molecules: A Quantum Theory (Clarendon, Oxford, 1994).

${ }^{8}$ J. Gaite, J. Margalef-Roig, and S. Miret-Artés, Phys. Rev. B 57, 13527 (1998).

${ }^{9}$ J. Gaite, J. Margalef-Roig, and S. Miret-Artés, Phys. Rev. B 59, 8593 (1999).
${ }^{10}$ A. L. Glebov, S. Miret-Artés, J. P. Toennies, F. Traeger, and J. Margalef-Roig, Phys. Rev. B 60, 6529 (1999).

${ }^{11}$ A. Böhm, Quantum Mechanics (Springer-Verlag, Berlin, 1979), p. 433.

${ }^{12}$ G. Benedek, R. Gerlach, A. Glebov, S. Miret-Artés, J. G. Skofronic, and J. P. Toennies, Phys. Rev. B 53, 11211 (1996).

${ }^{13}$ M. F. Bertino, S. Miret-Artés, J. P. Toennies, and G. Benedek, Phys. Rev. B 56, 9964 (1997); M. F. Bertino, S. Miret-Artés, and J. P. Toennies, Chem. Phys. Lett. 287, 663 (1998).

${ }^{14}$ M. Hernández, S. Miret-Artés, P. Villarreal, and G. DelgadoBarrio, Surf. Sci. 274, 21 (1992); S. Miret-Artés, ibid. 294, 141 (1993); 339, 205 (1995).

${ }^{15}$ G. Benedek and S. Miret-Artés, Surf. Sci. 339, L935 (1995).

${ }^{16}$ S. Miret-Artés, Surf. Sci. 366, L735 (1996).

${ }^{17}$ J. R. Taylor, Scattering Theory (Wiley, New York, 1972), p. 411.

${ }^{18}$ S. Miret-Artés, Phys. Rev. B 60, 1547 (1999). 